\title{
THE NON-ABELIAN TENSOR PRODUCT OF GROUPS AND RELATED CONSTRUCTIONS
}

\author{
by N. D. GILBERT $\dagger$ and P. J. HIGGINS
}

(Received 20 July, 1987)

Introduction. The tensor product of two arbitrary groups acting on each other was introduced by R. Brown and J.-L. Loday in $[5,6]$. It arose from consideration of the pushout of crossed squares in connection with applications of a van Kampen theorem for crossed squares. Special cases of the product had previously been studied by A. S.-T. Lue [10] and R. K. Dennis [7]. The tensor product of crossed complexes was introduced by R. Brown and the second author [3] in connection with the fundamental crossed complex $\pi(\mathbf{X})$ of a filtered space $\mathbf{X}$, which also satisfies a van Kampen theorem. This tensor product provides an algebraic description of the crossed complex $\pi(\mathbf{X} \otimes \mathbf{Y})$ and gives a symmetric monoidal closed structure to the category of crossed complexes (over groupoids). Both constructions involve non-abelian bilinearity conditions which are versions of standard identities between group commutators. Since any group can be viewed as a crossed complex of rank 1 , a close relationship might be expected between the two products. One purpose of this paper is to display the direct connections that exist between them and to clarify their differences.

Given a group $A$, we denote by $\mathbf{A}$ the crossed complex which has $A$ in dimension 1 but is otherwise trivial. For two arbitrary groups $A$ and $B$, without actions, the tensor product $\mathbf{A} \otimes \mathbf{B}$, as defined in [3], can be easily described; it is effectively the crossed module $A \square B \rightarrow A * B$, where $A \square B$ is the Cartesian subgroup of $A * B$ (the kernel of the canonical homomorphism $A * B \rightarrow A \times B$ ).

The tensor product $G \otimes H$ of two groups acting on one another is more subtle. It is a quotient of $G \square H$ and is a crossed module over a group $G \bowtie H$ introduced by $\mathrm{J}$. H. C. Whitehead [11] which we here call the Peiffer product of $G$ and $H$ (because of its connection with Peiffer identities). The tensor product $G \otimes H$ does not have the functorial properties enjoyed by the tensor product of crossed complexes but it essentially includes $\mathbf{A} \otimes \mathbf{B}$ as a special case: if $A$ and $B$ are groups without actions then $\mathbf{A} \otimes \mathbf{B}$ is the crossed module $\bar{A} \otimes \bar{B} \rightarrow \bar{A} \bowtie \bar{B}$ where $\bar{A}$ and $\bar{B}$ are obtained by freely generating from $A$ and $B$ two groups acting compatibly on each other.

On the other hand, for groups $G, H$ acting on each other, the crossed module $G \otimes H \rightarrow G \bowtie H$ cannot in general be written in the form $\mathbf{A} \otimes \mathbf{B}$ since the latter is always infinite, whereas $G \otimes H$ and $G \bowtie H$ are finite whenever $G$ and $H$ are finite [8]. We compute some examples which show that reasonable conjectures on how to obtain $G \otimes H$ from $\mathbf{G} \otimes \mathbf{H}$ are false.

1. Two tensor products of groups. A group $G$ may be regarded as a crossed complex $\mathbf{G}$ of rank 1; thus $\mathbf{G}$ has $G$ in dimension 1 and is otherwise trivial. Given two groups $G$ and $H$, we may therefore form the tensor product of the crossed complexes $\mathbf{G}$

$\dagger$ Research supported by the Science and Engineering Research Council under grant No. GR/D/2052.6.

Glasgow Math. J. 31 (1988) 17-29. 
and $\mathbf{H}$, as defined in [3]. This product is a crossed complex of rank 2, that is a crossed module, and it has an explicit description which we now recall (cf. [3, Proposition 6.1]).

For arbitrary groups $G, H$ we denote by $G \square H$ the Cartesian subgroup of the free product $G * H$ (that is, the kernel of the canonical homomorphism $G * H \rightarrow G \times H$ ).

1.1 Proposition. Given arbitrary groups $G$ and $H$, the tensor product of the crossed complexes $\mathbf{G}$ and $\mathbf{H}$ is the crossed module $i: G \square H \rightarrow G * H$ where $i$ is the inclusion map.

Now suppose that $G$ and $H$ act on each other on the right. Then the free product $G * H$ acts on both $G$ and $H$, each group acting on itself by conjugation. We assume that the actions are compatible, that is

$$
g_{1}^{\left(h^{8}\right)}=g_{1}^{g^{-1} h g} \quad h_{1}^{\left(g^{h}\right)}=h_{1}^{h^{-1} g h}
$$

for all $g, g_{1} \in G, h, h_{1} \in H$. The non-abelian tensor product of $G$ and $H$ was defined in $[5,6]$; after a change to right-handed notation it is the group $G \otimes H$ generated by symbols $g \otimes h(g \in G, h \in H)$ subject to defining relations

$$
\begin{aligned}
& g_{1} g \otimes h=\left(g_{1}^{8} \otimes h^{g}\right)(g \otimes h), \\
& g \otimes h_{1} h=(g \otimes h)\left(g^{h} \otimes h_{1}^{h}\right) .
\end{aligned}
$$

We note that these relations have the form of standard commutator identities when $g \otimes h$ is replaced by $[g, h]=g^{-1} h^{-1} g h$ and the actions by conjugation.

1.2 Lemma. (i) $G * H$ acts on $G \otimes H$ with

$$
(g \otimes h)^{w}=g^{w} \otimes h^{w}, \quad(w \in G * H) .
$$

(ii) $G \otimes H$ is the $G * H$-group generated by symbols $g \otimes h$ with defining relations

$$
\begin{gathered}
g_{1} g \otimes h=\left(g_{1} \otimes h\right)^{g}(g \otimes h), \\
g \otimes h_{1} h=(g \otimes h)\left(g \otimes h_{1}\right)^{h}, \\
(g \otimes h)^{w}=g^{w} \otimes h^{w} .
\end{gathered}
$$

Proof. It is straightforward to check that the subgroup of the free group on symbols $g \otimes h$ generated by the relators corresponding to the relations (1) and (2) admits the action of $G * H$ given by $(g \otimes h)^{w}=g^{w} \otimes h^{w}$. Note that compatibility is crucial here. This proves (i) and then (ii) is immediate.

1.3 Lemma. In $G \otimes H$ the following relations hold and are equivalent to (1) and (2):

$$
\begin{aligned}
& g g_{0} \otimes h=\left(g_{0} \otimes h^{g}\right)(g \otimes h), \\
& g \otimes h h_{0}=(g \otimes h)\left(g^{h} \otimes h_{0}\right) .
\end{aligned}
$$

Proof. Setting $g_{1}=g g_{0} g^{-1}$ and $h_{1}=h h_{0} h^{-1}$ in (1) and (2) yields (6) and (7).

We now have the two tensor products: however, the notation $G \otimes H$ will always refer to the non-abelian tensor product of Brown and Loday. In Proposition 1.1 we 
obtained a crossed module as a tensor product of crossed complexes. We now show that $G \otimes H$ is naturally a crossed module and to do this we need to introduce another product of pairs of groups and actions.

Given $G$ and $H$ acting compatibly on each other, we define their Peiffer product $G \bowtie H$ as the quotient of $G * H$ by the normal closure $K$ of all elements of the form

$$
h^{-1} g^{-1} h g^{h} \text { or } g^{-1} h^{-1} g h^{g}
$$

where $g \in G$ and $h \in H$ (see [11, p. 428]). Compatibility ensures that these elements act trivially on $G$ and $H$ so that $G \bowtie H$ acts on $G$ and $H$. Moreover, the canonical maps

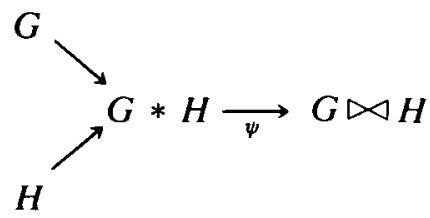

make $G$ and $H$ into crossed $G \bowtie H$-modules and the original actions are induced by the action of $G \bowtie H$.

1.4 Proposition. The tensor product $G \otimes H$ is a crossed $G \bowtie H$-module and there is a morphism $(\phi, \psi)$ of crossed modules

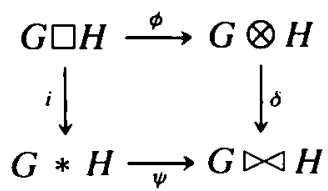

in which $\psi$ is the quotient map and $\phi([g, h])=g \otimes h$. Here $\delta(g \otimes h)=\psi([g, h])$ and the action of $G \bowtie H$ on $G \otimes H$ is induced by that of $G * H$. Furthermore, $L=\operatorname{ker} \phi$ is the normal closure in $G \square H$ of all elements

$$
w^{-1}[g, h] w\left[g^{w}, h^{w}\right]^{-1}
$$

where $w \in G * H$, and is a normal subgroup of $G * H$.

Proof. The group $G \square H$ is freely generated by all elements $[g, h], g \in G, h \in H$, $g \neq 1, h \neq 1$ (see [9]). There is therefore a morphism of groups $\phi: G \square H \rightarrow G \otimes H$ with $\phi([g, h])=g \otimes h$ for all $g \in G, h \in H$. Now $G * H$ acts on $G \square H$ by conjugation and on $G \otimes H$ by the action described in Lemma 1.2. The map $\phi$ is compatible with these actions because, for $g, g_{1} \in G$ and $h, h_{1} \in H$,

$$
\begin{aligned}
\phi\left(g^{-1}\left[g_{1}, h\right] g\right) & =\phi\left(\left[g_{1} g, h\right][g, h]^{-1}\right) \\
& =\left(g_{1} g \otimes h\right)(g \otimes h)^{-1} \\
& =\left(g_{1} \otimes h\right)^{g}
\end{aligned}
$$

and similarly $\phi\left(h^{-1}\left[g, h_{1}\right] h\right)=\left(g \otimes h_{1}\right)^{h}$. It follows that $L=$ ker $\phi$ is a normal subgroup of $G * H$. Furthermore, for $w \in G * H$, the element $u=w^{-1}[g, h] w\left[g^{w}, h^{w}\right]^{-1}$ is in $L$ since 
$\phi(u)=\phi([g, h])^{w} \phi\left(\left[g^{w}, h^{w}\right]\right)^{-1}=(g \otimes h)^{w}\left(g^{w} \otimes h^{w}\right)^{-1}=1$. However, by definition of $G \otimes H, L$ is generated, as a normal subgroup of $G \square H$, by all elements

and

$$
\left[g_{1} g, h\right][g, h]^{-1}\left[g^{-1} g_{1} g, h^{g}\right]^{-1}=g^{-1}\left[g_{1}, h\right] g\left[g^{-1} g_{1} g, h^{g}\right]^{-1}
$$

$$
[g, h]^{-1}\left[g, h_{1} h\right]\left[g^{h}, h^{-1} h_{1} h\right]^{-1}=h^{-1}\left[g, h_{1}\right] h\left[g^{h}, h^{-1} h_{1} h\right]^{-1} .
$$

Since these elements are of the form $w^{-1}[g, h] w\left[g^{w}, h^{w}\right]^{-1}$, the last part of the proposition follows.

Now, modulo $K=\operatorname{ker} \psi$, we have $g^{w}=w^{-1} g w$ and $h^{w}=w^{-1} h w$ so $L \subseteq K$ and $\psi(L)=1$. Consequently, there is a unique morphism $\delta: G \otimes H \rightarrow G \bowtie H$ with $\delta \phi=\psi i$, that is $\delta(g \otimes h)=\psi([g, h])$. It remains only to show that $K$ acts trivially on $G \otimes H$, for then $\psi$ induces an action of $G \bowtie H$ on $G \otimes H$ and it is immediate that $\delta: G \otimes H \rightarrow G \bowtie H$ is a crossed module and $(\phi, \psi)$ is a morphism of crossed modules. However, $K$ is normally generated by all $w=h^{-1} g^{-1} h g^{n}$ and $w^{\prime}=g^{-1} h^{-1} g h^{8}$ so it is enough to show that $w$ and $w^{\prime}$ act trivially on $G \otimes H$. This follows from the fact, already noted, that they act trivially on $G$ and $H$.

Let $A$ and $B$ be groups, with no actions assumed. We shall show how to construct the tensor product of the crossed complexes $\mathbf{A}$ and $\mathbf{B}$ as a crossed module $G \otimes H \rightarrow G \bowtie H$ by judicious choices of $G$ and $H$.

Define $\bar{A}$ to be the universal $B$-group on $A$, that is, $\bar{A}$ is the group generated by symbols $a^{b}(a \in A, b \in B)$ with defining relations $\left(a_{1} a_{2}\right)^{b}=a_{1}^{b} a_{2}^{b}$. Thus $\bar{A}$ is the free product of copies $A^{b}$ of $A$, one for each $b \in B$, and $B$ permutes these copies according to $\left(a^{b}\right)^{b^{\prime}}=a^{b b^{\prime}}$. We identify $A$ with the subgroup $A^{1}$ of $\bar{A}$ and so we write $a=a^{1}$. Similarly we define $\bar{B}$ to be the universal $A$-group on $B$.

The action of $B$ on $\bar{A}$ can be extended to an action of $\bar{B}$ on $\bar{A}$ by the rule $\left(a_{1}^{b^{\prime}}\right)^{b^{a}}=a_{1}^{b^{\prime} a^{-1} b a}$ where $a$ is identified with $a^{1}$ and acts by conjugation in $\bar{A}$. Thus, in normal form,

$$
\left(a_{1}^{b^{\prime}}\right)^{b^{a}}=a^{-1} a^{b} a_{1}^{b^{\prime} b}\left(a^{b}\right)^{-1} a .
$$

Similarly we can define an action of $\bar{A}$ on $\bar{B}$ and it is easy to see that the two actions are compatible.

We may now form $\bar{A} \otimes \bar{B}$ : it is a crossed module over $\bar{A} \bowtie \bar{B}$.

1.5 Proposition. The crossed module $\bar{A} \otimes \bar{B} \rightarrow \bar{A} \bowtie \bar{B}$ is isomorphic to the tensor product of $A$ and $B$ regarded as crossed complexes of rank 1 , that is, to the crossed module $A \square B \rightarrow A * B$.

Proof. Consider the composite morphism of crossed modules where $i, j, k, l$ are inclusion maps and $(\phi, \psi)$ is the morphism given by Proposition 1.4 applied to $\bar{A}$ and $\bar{B}$.

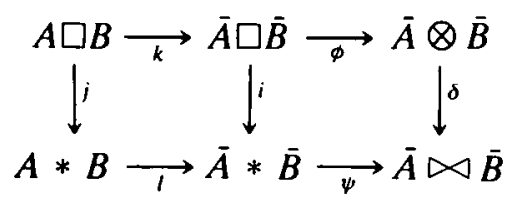


The kernel $K$ of $\psi$ is the normal subgroup of $\bar{A} * \bar{B}$ generated by all $x^{-1} y^{-1} x y^{x}$ and $y^{-1} x^{-1} y x^{y}$ for $x \in \bar{A}, y \in \bar{B}$. Modulo this kernel we have $a^{b} \equiv b^{-1} a b$ and $b^{a} \equiv a^{-1} b a$ for $a \in A, b \in B$. So $\bar{A} * \bar{B}$ is generated by $A * B$ and $K$. Since $\psi$ is surjective, it follows that $\psi l$ is surjective.

On the other hand, because of the freeness of $\bar{A}$ and $\bar{B}$, there is a morphism $\theta: \bar{A} * \bar{B} \rightarrow A * B$ with

$$
\theta\left(a^{b}\right)=b^{-1} a b, \quad \theta\left(b^{a}\right)=a^{-1} b a \text { for } a \in A, \quad b \in B .
$$

Clearly $\theta l$ is the identity on $A * B$. Also, if $x \in \bar{A}$, one may deduce from (9) firstly that $\theta\left(x^{b}\right)=b^{-1} \theta(x) b$ and then that $\theta\left(x^{b^{a}}\right)=\theta\left(b^{a}\right)^{-1} \theta(x) \theta\left(b^{a}\right)$, where $a \in A$ and $b \in B$. Hence, for all $x \in \bar{A}$ and $y \in \bar{B}$ we have $\theta\left(x^{y}\right)=\theta(y)^{-1} \theta(x) \theta(y)$ and similarly $\theta\left(y^{x}\right)=$ $\theta(x)^{-1} \theta(y) \theta(x)$. Thus $\theta(K)=1$. If now, $u \in A * B$ and $\psi l(u)=1$, then $l(u) \in K$ and therefore $u=\theta l(u)=1$. This proves that $\psi l: A * B \rightarrow \bar{A} \bowtie \bar{B}$ is an injection and therefore an isomorphism.

Now consider $\phi k: A \square B \rightarrow \bar{A} \otimes \bar{B}$. It is clearly an injection, because $\delta \phi k=\theta l j$ is an injection. Thus the theorem will be proved if we can show that $\phi k$ is a surjection. This we will do by showing that $\bar{A} \otimes \bar{B}$ is generated as crossed $\bar{A} \bowtie \bar{B}$-module by the elements $\phi k([a, b])=a \otimes b$. Since the action of $\bar{A} \bowtie \bar{B}$ on $\bar{A} \otimes \bar{B}$ is induced from that $\bar{A} * \bar{B}$, this is equivalent to showing that $\bar{A} \otimes \bar{B}$ is generated as $\bar{A} * \bar{B}$-group by all $a \otimes b$ with $a \in A, b \in B$. Now $\bar{A} \otimes \bar{B}$ is certainly generated as a group by all $x \otimes y, x \in \bar{A}, y \in \bar{B}$ and the relations (3), (4) can be used to express any such $x \otimes y$ as a product of elements of the form $\left(a_{1}^{b} \otimes b_{1}^{a}\right)^{w}$, where $a, a_{1} \in A, b, b_{1} \in B$ and $w \in \bar{A} * \bar{B}$. Finally,

$$
\begin{aligned}
a_{1}^{b} \otimes b_{1}^{a} & =\left(a_{1} \otimes b\right)^{-1}\left(a_{1} \otimes b b_{1}^{a}\right) \\
& =\left(a_{1} \otimes b\right)^{-1}\left(a_{1} \otimes b_{1}^{a}\right)\left(a_{1} \otimes b\right)^{b_{1}^{a}} \\
& =\left(a_{1} \otimes b\right)^{-1}\left(a \otimes b_{1}\right)^{a_{1}}\left(a_{1} \otimes b_{1}\right)\left(a \otimes b_{1}\right)^{-1}\left(a_{1} \otimes b\right)^{b_{1}^{a}}
\end{aligned}
$$

and this completes the proof.

2. The Peiffer product. We shall return in Section 3 to the crossed module morphism of Proposition 1.4. Before doing so we consider the Peiffer product $G \bowtie H$ in more detail. As mentioned in the introduction, this construction was introduced by Whitehead in [11]. There he posed his famous question on the asphericity of subcomplexes of aspherical 2-complexes and reformulated it as part of the wider problem of finding conditions under which the groups $G$ and $H$ are embedded in $G \bowtie H$.

Let $G$ and $H$ be groups acting compatibly on each other and let $K$ be the kernel of the natural map $\psi: G * H \rightarrow G \bowtie H$. Then modulo $K, h g \equiv g h^{g}$, so that every element of $G \bowtie H$ can be written as $\psi(g) \psi(h)$ for suitable $g, h$. We write $\langle g, h\rangle$ for $\psi(g) \psi(h)$. By considering the implied presentation of $G \bowtie H$ as $(G * H) / K$ it is easy to see that the relations

$$
\langle g, h\rangle\left\langle g_{1}, h_{1}\right\rangle=\left\langle g g_{1}, h^{g_{1}} h_{1}\right\rangle=\left\langle g g_{1}^{h^{-1}}, h h_{1}\right\rangle
$$

are defining relations for $G \bowtie H$ on the generators $\langle g, h\rangle$ and so $G \bowtie H$ is a homomorphic image of both the semidirect products $G \ltimes H$ and $G \rtimes H$. This explains our choice of 
notation. The group $G \bowtie H$ is obtained from $G \ltimes H$ (or from $G \rtimes H$ ) by imposing the relations

$$
\left(g^{-1} g^{h}, 1\right)=\left(1, h^{-8} h\right) \text {. }
$$

These facts were proved by $\mathrm{R}$. Brown in [1].

Given two crossed $P$-modules $\lambda: G \rightarrow P$ and $\mu: H \rightarrow P$, we can form the Peiffer product $G \bowtie H$ using the actions of $G$ and $H$ on each other induced via $P$. Such actions are always compatible. R. Brown also proved in [1] that in this case $G \bowtie H$ is itself a crossed $P$-module with boundary map $\partial: G \bowtie H \rightarrow P$ given by $\langle g, h\rangle \mapsto \lambda(g) \mu(h)$ and is the coproduct of $G$ and $H$ in the category of crossed $P$-modules. The expression of $G \bowtie H$ as a quotient of $G \ltimes H$ greatly facilitates the study of the kernel of $\partial: G \bowtie H \rightarrow P$.

On the other hand, if $G$ and $H$ act compatibly on one another, then each is a crossed $G \bowtie H$-module with boundary map induced by the respective inclusion into $G * H$ and the given actions then coincide with those obtained via $G \bowtie H$. It follows that the coproduct of $G$ and $H$ as crossed $G \bowtie H$-modules is just the identity map $G \bowtie H \rightarrow G \bowtie H$.

We now consider some special cases in which the Peiffer product $G \bowtie H$ of groups $G$ and $H$ acting compatibly on one another may be described explicitly in terms of $G$ and $H$. We write $D_{H}(G)$ for the displacement subgroup of $G$ relative to the action of $H$, that is the subgroup of $G$ generated by all elements $g^{-1} g^{h}$ where $g \in G$ and $h \in H$. Then $D_{H}(G)$ is normal in $G$ and $G / D_{H}(G)$ is the largest quotient of $G$ on which $H$ acts trivially: we denote this quotient by $G_{H}$.

2.1 Proposition. Let $\lambda: G \rightarrow P$ and $\mu: H \rightarrow P$ be crossed $P$-modules such that $\lambda(G) \subseteq \mu(H)$ and suppose that $\mu: H \rightarrow \mu(H)$ is split by a homomorphism $\sigma: \mu(H) \rightarrow H$. Then the Peiffer product $G \bowtie H$ formed with respect to the actions of $G$ and $H$ on each other via $P$ is isomorphic as a group to $G_{H} \times H$.

Proof. Form the semidirect product $G \ltimes H$ and define a map $\xi: G \ltimes H \rightarrow G \times H$ by $(g, h) \mapsto(g, \sigma \lambda(g) h)$. Then $\xi$ is an isomorphism, for it is clearly bijective and

$$
\begin{aligned}
\xi\left(\left(g_{1}, h_{1}\right)(g, h)\right) & =\xi\left(g_{1} g, h_{1}^{g} h\right) \\
& =\left(g_{1} g, \sigma \lambda\left(g_{1} g\right) h_{1}^{g} h\right) \\
& =\left(g_{1} g, \sigma \lambda\left(g_{1}\right) \sigma \lambda(g) h_{1}^{g} h\right) .
\end{aligned}
$$

Now $h_{1}^{g}=h_{1}^{\lambda(g)}=h_{1}^{\mu(y)}$ for some $y \in H$ and $\sigma \lambda(g) \equiv y(\bmod \operatorname{ker} \mu)$. Hence $h_{1}^{g}=h_{1}^{\mu(y)}=$ $y^{-1} h_{1} y=\sigma \lambda(g)^{-1} h_{1} \sigma \lambda(g)$ since ker $\mu$ is central in $H$. So

$$
\xi\left(\left(g_{1}, h_{1}\right)(g, h)\right)=\left(g_{1} g, \sigma \lambda\left(g_{1}\right) h_{1} \sigma \lambda(g) h\right)=\xi\left(g_{1}, h_{1}\right) \xi(g, h) .
$$

Further, $\xi$ maps the relation (10) to

Now

$$
\left(g^{-1} g^{h}, \sigma \lambda\left(g^{-1} g^{h}\right)\right)=\left(1, h^{-g} h\right) .
$$

$$
\begin{aligned}
\sigma \lambda\left(g^{-1} g^{h}\right) & =\sigma([\lambda(g), \mu(h)]) \\
& =[\sigma \lambda(g), h] \quad \text { (since ker } \mu \text { is central) } \\
& =\sigma \lambda\left(g^{-1}\right) h^{-1} \sigma \lambda(g) h \\
& =h^{-g} h .
\end{aligned}
$$


So the kernel of the map $G \ltimes H \rightarrow G \bowtie H$ is mapped to the normal closure in $G \times H$ of the elements $\left(g^{-1} g^{h}, 1\right)$ and so $G \bowtie H \cong G_{H} \times H$.

Note that under the hypotheses of Proposition $2.1, G_{H}$ is abelian and since $H$ is a crossed module with a splitting of its boundary map, we have a split central extension

$$
0 \rightarrow \operatorname{ker} \mu \rightarrow H \rightarrow \mu(H) \rightarrow 1
$$

and $H \cong \operatorname{ker} \mu \times \mu(H)$ as groups. Further, if we can find a $P$-equivariant splitting $\sigma$ then this isomorphism and that of Proposition 2.1 are isomorphisms of crossed $\boldsymbol{P}$-modules.

2.2 Corollary. Under the hypotheses of Proposition 2.1 the canonical map $H \rightarrow$ $G \bowtie H$ is an embedding, but the canonical map $G \rightarrow G \bowtie H$ is an embedding if and only if $\operatorname{ker} \lambda \cap D_{H}(G)=1$.

Proof. Identifying $G \bowtie H$ with $G_{H} \times H$, the canonical maps in question are $g \mapsto$ $\left(g D_{H}(G), \sigma \lambda(g)\right)$ and $h \mapsto(1, h)$. So $H$ certainly embeds in $G \bowtie H$ and the statement for $G$ follows since $\sigma$ is injective.

2.3 Corollary. If $\lambda: G \rightarrow P$ is a crossed $P$-module then $G \bowtie P$ and $G_{P} \times P$ are isomorphic as crossed P-modules.

In particular, if $M$ is a normal subgroup of $P$ we can form the Peiffer product of $M$ and $P$ with respect to the conjugation actions and $M \bowtie P \cong M /[M, P] \times P$. So, putting $M=P$, we find $P \bowtie P \cong P^{\mathrm{ab}} \times P$.

We now return to the general case of groups $G$ and $H$ given as crossed $P$-modules acting on each other via $\lambda: G \rightarrow P$ and $\mu: H \rightarrow P$. The kernel of $\partial: G \bowtie H \rightarrow P$ has been investigated by $\mathrm{R}$. Brown in [1]. Let $G \times_{P} H$ be the pullback: this is again a crossed $P$-module under the diagonal action of $P$ with boundary map given by $\delta(g, h)=\lambda(g)=$ $\mu(h)$. It is easy to verify that in fact $G \times{ }_{P} H$ is the product of $G$ and $H$ in the category of crossed $P$-modules. Define the function $\xi: G \times H \rightarrow G \times{ }_{P} H$ by $\zeta(g, h)=\left(g^{-1} g^{h}, h^{-g} h\right)$ and let $J$ be the subgroup of $G \times{ }_{P} H$ generated by the image of $\zeta$. Then $J$ is normal in $G \times{ }_{P} H$ and contains the commutator subgroup. Let us write $M=\lambda(G)$ and $N=\mu(H)$ : then there are exact sequences of groups, [1, Propositions 2.5 and 2.8],

$$
\begin{gathered}
0 \rightarrow\left(G \times_{P} H\right) / J \rightarrow G \bowtie H \rightarrow P, \\
0 \rightarrow(\operatorname{ker} \lambda \oplus \operatorname{ker} \mu) \cap J \rightarrow \operatorname{ker} \lambda \oplus \operatorname{ker} \mu \rightarrow\left(G \times_{P} H\right) / J \rightarrow(M \cap N) /[M, N] \rightarrow 0,
\end{gathered}
$$

where the map $j$ in (11) is induced by the map $G \times{ }_{P} H \rightarrow G \ltimes H$ given by $(g, h) \mapsto$ $\left(g, h^{-1}\right)$. If $\lambda$ and $\mu$ are injective then (11) and (12) show that ker $\partial \cong(M \cap N) /[M, N]$. In particular, for any normal subgroups $M$ and $N$ of a group $P$, there is a short exact sequence

$$
0 \rightarrow \frac{M \cap N}{[M, N]} \rightarrow M \bowtie N \rightarrow M N \rightarrow 1
$$


showing how $M \bowtie N$ depends on the normal structure of $M$ and $N$ relative to each other. Note further that both $M$ and $N$ embed in $M \bowtie N$.

3. Induced crossed modules and the tensor products. We recall from [2] the definition of an induced crossed module. Suppose that $d: A \rightarrow P$ is a crossed $P$-module and that $f: P \rightarrow S$ is a homomorphism of groups. Then there is a crossed $S$-module $C=f_{*} A$ and a morphism of crossed modules

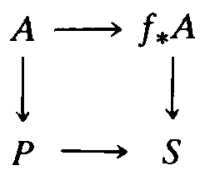

which is universal for morphisms from $A$ to crossed $S$-modules which induce $f: P \rightarrow S$. The crossed $S$-module $f_{*} A$ is said to be induced by $f$ and $f_{*}$ is a functor from crossed $P$-modules to crossed $S$-modules. A presentation of $f_{*} A$ is given in [2]. For our present purposes we have need only of the simpler description that applies when $f$ is surjective.

3.1 Proposition [2]. If $f: P \rightarrow S$ is a surjective homomorphism and $A$ is a crossed $P$-module then $f_{*} A=A_{\text {ker } f}$.

R. Brown has asked the following questions. Does the crossed module morphism of Proposition 1.4 present $G \otimes H$ as the crossed $G \bowtie H$-module induced from $G \square H$ by the natural map $\psi: G * H \rightarrow G \bowtie H$ ? Or is $G \otimes H$ the crossed $S$-module induced from $G \square H$ by some other morphism $G * H \rightarrow S$ ?

From Proposition 3.1 the induced crossed module $\psi_{*}(G \square H)$ is obtained from $G \square H$ by killing the action of $K=\operatorname{ker} \psi$. Since this action is by conjugation we have

$$
\psi_{*}(G \square H)=(G \square H) /[G \square H, K] .
$$

Let $\kappa: G \square H \rightarrow \psi_{*}(G \square H)$ be the natural map. By the universal property of induced crossed modules there is a morphism $\tau: \psi_{*}(G \square H) \rightarrow G \otimes H$ of crossed $G \bowtie H$-modules such that $\tau_{K}=\phi$. The question at issue now is whether or not $\tau$ is an isomorphism.

We consider the simplest case, in which $G$ and $H$ act on one another trivially. In this case $G \bowtie H$ is just the direct product $G \times H$ and $K=G \square H$. Thus $\psi_{*}(G \square H)$ is $(G \square H)^{\text {ab }}$ which is free abelian on the basis $\{[g, h] \mid g \neq 1, h \neq 1\}$ of mixed commutators in $G * H$, which we now wish to regard merely as a set of ordered pairs.

Since we are assuming that $G$ and $H$ act trivially on one another, from (6) we obtain the relation

$$
g g_{0} \otimes h=\left(g_{0} \otimes h\right)(g \otimes h) .
$$

Now $G \otimes H$ is abelian (it is a homomorphic image of $(G \square H)^{\mathrm{ab}}$ ) and so

Similarly

$$
g g_{0} \otimes h=(g \otimes h)\left(g_{0} \otimes h\right)=g_{0} g \otimes h .
$$

$$
g \otimes h h_{0}=(g \otimes h)\left(g \otimes h_{0}\right)=g \otimes h h_{0} .
$$


It follows that $G \otimes H \cong G^{\text {ab }} \otimes_{\mathbb{Z}} H^{\text {ab }}$ (see [6, Proposition 2.4]) and that $G * H$ and $G \times H$ act trivially on $G \otimes H$.

It is now clear that the map $\tau$ from $\psi_{*}(G \square H)=(G \square H)^{\mathrm{ab}}$ to $G \otimes H=G^{\mathrm{ab}} \otimes_{\mathbb{Z}} H^{\mathrm{ab}}$ is not an isomorphism unless one of $G, H$ is trivial; for if $g \in G, h \in H, g \neq 1, h \neq 1$, then $[g, h]\left[g^{-1}, h\right] \neq 1$ in $(G \square H)^{\text {ab }}$ but $(g \otimes h)\left(g^{-1} \otimes h\right)=1$ in $G \otimes H$. In fact it follows from Lemma 1.2 and Proposition 3.1 that $G \otimes H$ is obtained from $G \square H$ by killing the action of $G * H$, that is:

3.2 Proposition. If $G$ and $H$ act trivially on each other, $G \otimes H$ is the crossed module over the trivial group induced from $G \square H \rightarrow G * H$ by the map $G * H \rightarrow 1$.

Thus there remains the possibility that $G \otimes H$ is in all cases the crossed module induced from $G \square H \rightarrow G * H$ by some quotient map $\chi: G * H \rightarrow S$. We show that this is not the case by means of the example of two infinite cyclic groups acting non-trivially on each other.

3.3 Proposition. Let $X$ and $Y$ be infinite cyclic groups generated by $x$ and $y$ respectively, acting on each other by

$$
x^{y}=x^{-1}, \quad y^{x}=y^{-1} .
$$

These actions are compatible and the Peiffer product $X \bowtie Y$ is the quaternion group $Q=\{ \pm 1, \pm i, \pm j, \pm k\}$ with canonical map $\psi: X * Y \rightarrow Q$ given by $\psi(x)=i$ and $\psi(y)=j$. The induced crossed $Q$-module $\psi_{*}(X \square Y)$ is $\mathbb{Z}^{4}$ and $X \otimes Y$ is $\mathbb{Z}^{2}$ with bases, $Q$-actions and boundary maps given by the formulae (13), . . , (17) and (20), . . , (24) below.

Proof. The compatibility of the given actions is easily checked. Further, it is clear that if we are given presentations of groups $G$ and $H$ that act compatibily on one another, we obtain a presentation of $G \bowtie H$ by adjoining to the natural presentation of $G * H$ the relations (8) between generators. Hence

$$
X \bowtie Y=\left\langle x, y \mid y^{-1} x y=x^{-1}, x^{-1} y x=y^{-1}\right\rangle .
$$

The map $x \mapsto i, y \mapsto j$ defines a surjection $X \bowtie Y \rightarrow Q$. However, in $X \bowtie Y,[x, y]=x^{-2}$ and $[y, x]=y^{-2}$ and so $x^{2}=y^{-2}=[y, x]$. Hence

$$
y x=x y[y, x]=x y^{-1}=x^{3} y .
$$

Therefore $x=y^{-1} x^{3} y=x^{-3}$ and so $x^{4}=1$ and since $x^{2}=y^{-2}, y^{4}=1$. Every element of $X \bowtie Y$ can now be written as $x^{r} y^{s}$ where $r, s=0,1,2,3$ and $x^{2} y^{2}=1$. This implies $|X \bowtie Y| \leq 8$ and so $X \bowtie Y \cong Q$.

We now compute $\psi_{*}(X \square Y)$ where $\psi: X * Y \rightarrow Q$ is given by $x \mapsto i, y \mapsto j$. Let $K=\operatorname{ker} \psi, M=\psi_{*}(X \square Y), \phi: X \square Y \rightarrow M$ and $N=\operatorname{ker}(\delta: M \rightarrow Q)$. For each generator $\left[x^{m}, y^{n}\right]$ of $X \square Y$ we have

$$
\psi\left(\left[x^{m}, y^{n}\right]\right)=\left[i^{m}, j^{n}\right]=(-1)^{m n}
$$


Thus $\delta(M)=\{ \pm 1\}$ and $N$ is central in $M$ and of index 2: hence $M$ is abelian and $\delta(M)$ acts trivially on $M$. So $i^{2}, j^{2}$ and $k^{2}$ act trivially on $M$. We write $\left\{x^{m}, y^{n}\right\}$ for $\phi\left(\left[x^{m}, y^{n}\right]\right)$. Now

$$
\begin{aligned}
\left\{x^{m}, y^{n}\right\} & =\left\{x^{m}, y^{n}\right\}^{i^{2}} \\
& =\phi\left(\left[x^{m}, y^{n}\right]^{x^{2}}\right) \\
& =\phi\left(\left[x^{m+2}, y^{n}\right]\left[x^{2}, y^{n}\right]^{-1}\right) \\
& =\left\{x^{m+2}, y^{n}\right\}\left\{x^{2}, y^{n}\right\}^{-1}
\end{aligned}
$$

So $\left\{x^{m+2}, y^{n}\right\}=\left\{x^{m}, y^{n}\right\}\left\{x^{2}, y^{n}\right\}$ and similarly $\left\{x^{m}, y^{n+2}\right\}=\left\{x^{m}, y^{n}\right\}\left\{x^{m}, y^{2}\right\}$. Thus $M$ is generated as a group by the four elements

$$
\{x, y\},\left\{x^{2}, y\right\},\left\{x, y^{2}\right\},\left\{x^{2}, y^{2}\right\}
$$

The actions of $i$ and $j$ on these generators are easily computed and we find

$$
\begin{gathered}
\{x, y\}^{i}=\left\{x^{2}, y\right\}\{x, y\}^{-1}, \quad\{x, y\}^{j}=\left\{x, y^{2}\right\}\{x, y\}^{-1}, \\
\left\{x^{2}, y\right\}^{i}=\left\{x^{2}, y\right\}, \quad\left\{x^{2}, y\right\}^{j}=\left\{x^{2}, y^{2}\right\}\left\{x^{2}, y\right\}^{-1}, \\
\left\{x, y^{2}\right\}^{i}=\left\{x^{2}, y^{2}\right\}\left\{x, y^{2}\right\}^{-1}, \quad\left\{x, y^{2}\right\}^{j}=\left\{x, y^{2}\right\}, \\
\left\{x^{2}, y^{2}\right\}^{i}=\left\{x^{2}, y^{2}\right\}, \quad\left\{x^{2}, y^{2}\right\}^{j}=\left\{x^{2}, y^{2}\right\} .
\end{gathered}
$$

So $M$, as a $Q$-module, is a homomorphic image of $\mathbb{Z}^{4}$ with basis $b_{1}, b_{2}, b_{3}, b_{4}$ mapping respectively to $\{x, y\},\left\{x^{2}, y\right\},\left\{x, y^{2}\right\},\left\{x^{2}, y^{2}\right\}$ and with the action of $i$ and $j$ given by

$$
\begin{gathered}
b_{1}^{i}=b_{2}-b_{1}, \quad b_{1}^{j}=b_{3}-b_{1}, \\
b_{2}^{i}=b_{2}, \quad b_{2}^{j}=b_{4}-b_{2}, \\
b_{3}^{i}=b_{4}-b_{3}, \quad b_{3}^{j}=b_{3}, \\
b_{4}^{i}=b_{4}, \quad b_{4}^{j}=b_{4} .
\end{gathered}
$$

It is easy to verify that the map $d: \mathbb{Z}^{4} \rightarrow Q$ given by

$$
b_{1} \mapsto-1, \quad b_{r} \mapsto 1 \quad(r \geq 2),
$$

is a crossed module and that the map $\theta: X \square Y \rightarrow \mathbb{Z}^{4}$ given by

$$
\begin{gathered}
\theta\left(\left[x^{2 r}, y^{2 s}\right]\right)=r s b_{4}, \\
\theta\left(\left[x^{2 r+1}, y^{2 s}\right]\right)=s b_{3}+r s b_{4}, \\
\theta\left(\left[x^{2 r}, y^{2 s+1}\right]\right)=r b_{2}+r s b_{4}, \\
\theta\left(\left[x^{2 r+1}, y^{2 s+1}\right]\right)=b_{1}+r b_{2}+s b_{3}+r s b_{4},
\end{gathered}
$$


gives a morphism of crossed modules

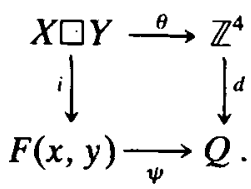

By the universal property of $\delta: M \rightarrow Q$ there is a morphism of crossed modules

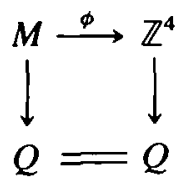

and it follows that $M$ is isomorphic as a crossed $Q$-module to $\mathbb{Z}^{4}$.

Now $X \otimes Y$ is abelian (since $M$ is) and in $X \otimes Y$,

$$
\begin{gathered}
x \otimes y^{2}=(x \otimes y)\left(x^{-1} \otimes y\right), \\
x \otimes y=x^{-1} x^{2} \otimes y=\left(x^{-1} \otimes y\right)\left(x^{2} \otimes y\right),
\end{gathered}
$$

and so

$$
\left(x \otimes y^{2}\right)\left(x^{2} \otimes y\right)=(x \otimes y)^{2} .
$$

Further, $x \otimes y^{2}=x^{-1} x^{2} \otimes y^{2}=\left(x^{-1} \otimes y^{2}\right)\left(x^{2} \otimes y^{2}\right)$ so that

But also

$$
x^{-1} \otimes y^{2}=\left(x \otimes y^{2}\right)\left(x^{2} \otimes y^{2}\right)^{-1} \text {. }
$$

Therefore

$$
\begin{aligned}
x^{-1} \otimes y^{2} & =\left(x \otimes y^{2}\right)^{y}=(x \otimes y)^{-1}\left(x \otimes y^{3}\right) \\
& =(x \otimes y)^{-1}\left(x \otimes y^{2}\right)(x \otimes y) \\
& =x \otimes y^{2} .
\end{aligned}
$$

$$
x^{2} \otimes y^{2}=1 \text {. }
$$

(18) and (19) show that $x \otimes y$ and $x^{2} \otimes y$ generate $X \otimes Y$ as an abelian group and the action of $Q$ is given by

$$
\begin{gathered}
(x \otimes y)^{i}=x \otimes y^{-1}=\left(x^{2} \otimes y\right)(x \otimes y)^{-1}, \\
(x \otimes y)^{j}=x^{-1} \otimes y=\left(x^{2} \otimes y\right)^{-1}(x \otimes y), \\
\left(x^{2} \otimes y\right)^{i}=x^{2} \otimes y^{-1}=x^{2} \otimes y, \\
\left(x^{2} \otimes y\right)^{j}=x^{-2} \otimes y=\left(x^{2} \otimes y\right)^{-1} .
\end{gathered}
$$

It is now straightforward to show that $\mathbb{Z}^{2}$, with basis

$$
x \otimes y, \quad x^{2} \otimes y
$$

and the action just given, is a crossed $Q$-module and that the defining relations for $X \otimes Y$ are satisfied. 
As a consequence of this computation, we see that in the diagram

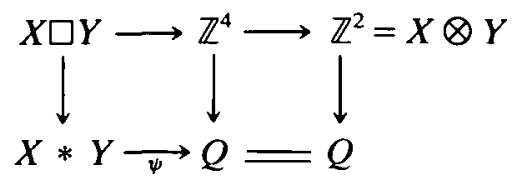

the only elements of $Q$ which act trivially on $X \otimes Y$ are \pm 1 , and these already act trivially on $\mathbb{Z}^{4}$. Therefore, all elements of $X * Y$ which act trivially on $X \otimes Y$ already act trivially on $\mathbb{Z}^{4}$ and it follows that $X \otimes Y$ cannot be obtained from $X \square Y$ by killing the action of a normal subgroup of $X * Y$. Thus $X \otimes Y$ is not the crossed $S$-module induced from $X \square Y \rightarrow X * Y$ by any surjection $X * Y \rightarrow S$. It seems to be difficult (but less interesting) to determine when $X \otimes Y$ is induced by a non-surjective map $X * Y \rightarrow S$.

We conclude with a discussion of another special case of the tensor product. Given any group $G$ we may form its tensor square $G \otimes G$ using the conjugation action of $G$ on itself. Then $G \otimes G$ is a $G * G$-group and the images $g_{1}$ and $g_{2}$ of $g \in G$ in the factors of $G * G$ each act via

$$
(x \otimes y)^{g_{i}}=g^{-1} x g \otimes g^{-1} y g \quad(i=1,2) .
$$

It follows that the kernel of the folding map $G * G \rightarrow G$ which identifies the two copies of $G$ in $G * G$ acts trivially on $G \otimes G$ and that $G \otimes G$ is a crossed $G$-module with $G$-action given by $(x \otimes y)^{g}=g^{-1} x g \otimes g^{-1} y g$ and boundary map $\delta: G \otimes G \rightarrow G$ by $x \otimes y \mapsto[x, y]$. We refer to [4] and [6] for further results on and applications of the tensor square.

The question of the relationship between $G \square G$ and $G \otimes G$ first arose in conversations between $\mathrm{H}$. J. Baues and R. Brown. We shall show that $G \otimes G$ is not induced from the inclusion map $G \square G \rightarrow G * G$ by the folding map $G * G \rightarrow G$.

Let $(G, G)$ denote the induced crossed module just described. Then $(G, G)$ is obtained from $G \square G$ by killing the action of the kernel of the folding map, that is by making the two images of $g \in G$ in $G * G$ act in the same way. It follows that $(G, G)$ is the group generated by all pairs $(x, y)$ where $x, y \in G$, subject to defining relations

$$
\begin{gathered}
(1, x)=1=(x, 1), \\
(x, y)(x y, z)=(x, z y)(y, z) .
\end{gathered}
$$

The $G$-action is given by

$$
(x, y)^{g}=(x g, y)(g, y)^{-1}=(x, g)^{-1}(x, y g)
$$

and the boundary map is $d:(x, y) \mapsto[x, y]$. So if $G$ is abelian, $d$ is the zero map and $(G, G)$ is abelian. It is easy to see that if $G$ is cyclic of order 2 then $(G, G)$ is infinite cyclic, whereas $G \otimes G \cong G$.

\section{REFERENCES}

1. R. Brown, Coproducts of crossed $P$-modules: applications to second homotopy groups and to the homology of groups, Topology 23 (1984), 337-345. 
2. R. Brown and P. J. Higgins, On the connection between the second relative homotopy groups of some related spaces, Proc. London Math. Soc. (3) 36 (1978), 193-212.

3. R. Brown and P. J. Higgins, Tensor products and homotopies for $\omega$-groupoids and crossed complexes, J. Pure Appl. Algebra 47 (1987), 1-33.

4. R. Brown, D. L. Johnson and E. F. Robertson, Some computations of non-abelian tensor products of groups, J. Algebra 111 (1987), 177-202.

5. R. Brown and J.-L. Loday, Excision homotopique en basse dimension, C.R. Acad. Sci. Paris Sér. I Math. 298 (1984), 353-356.

6. R. Brown and J.-L. Loday, Van Kampen theorems for diagrams of spaces, Topology 26 (1987), 311-335.

7. R. K. Dennis, In search of new homology functors having a close relationship to $K$-theory preprint (Cornell Univ., 1976).

8. G. J. Ellis, The non-abelian tensor product of finite groups is finite, J. Algebra 111 (1987), 203-205. $136-144$.

9. F. W. Levi, The commutator group of a free product, J. Indian Math. Soc. (N.S.) 4 (1940), 309-312.

10. A. S.-T. Lue, The Ganea map for nilpotent groups, J. London Math. Soc. (2) 14 (1976),

11. J. H. C. Whitehead, On adding relations to homotopy groups, Ann. of Math. (2) 42 (1941), 409-428.

N. D. Gilbert

Department of Pure Mathematics

UNiversity College of NorTh Wales

BANGOR

GWYNEDD LL57 2UW

P. J. Higgins

Department of Mathematical Sciences

UNIVERSITY OF DURHAM

SCIENCE LABORATORIES

SOUTH ROAD

Durham DH1 3LE 